\title{
Calcul des pertes d'énergie dans un estuaire à marée (Gironde) Principe et exécution du calcul à l'aide d'une machine mathématique
}

\section{Calculation of energy losses in a tidal estuary (Gironde) Theory and accomplishment of calculation by means of an electronic calculator}

\author{
PAR M. FAURE,
}

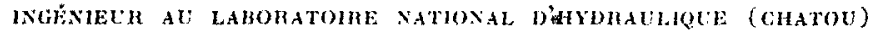

\begin{abstract}
Les équations aux dérivées partielles (équation de continuité et équalion dynamique) sont résolues numeriquement en remplacant les dérivées ptr des différences finies. La division de l'estuaire de la Gironde en pingt troncons et du cycle des mares en cinquante intervalles, conduit $\dot{a}$ 90.000 opérations exécutées mécaniquement en quelques dizaines d'heures, avec une précision quit, en génértal, ne dépend que de la précision des données et $d u$ déconpage en troncons et en intervalles. La machine donne, d'ailleurs, $n$ n certain nombre de résultats intermédiaires inté. ressants (cubature, bitesses, accólérations, efos). La perte locale d'énergie croit lorsque l'estuaire se rétrécit.
\end{abstract}

Partial differential equations (equation of continuity and dynamic equation) are solved numerically by substituting finite differences for the differentials. Dibision of the fironde esturery inte 20 sections, and diotsion of the fide rycle into 50 internals, inuolves 90,000 merhanical operations in about thirfy hours; the acuraty obtained generally tepends only on the aceuracy of the data and of the division into sections and intervals. In addition, the machine gives a certain number of interesting intermediury results (cubalure, pelocities, aceleralion, ela.). The local loss of energy increases when the estuary nurrows.

\section{I. - INTRODUCTION}

On admet généralement que les équations du mouvement non permanent régissent la propagation de l'onde de marée dans un estuaire. Connaissant sa géomćtrie et les parametres qui fixent les pertes de charge, il est possible de traiter le problème numériquement, ce que firent des ingénieurs belges ou nécrlandais [1]. Dans leurs calculs, les paramitres fournissant les valeurs des pertes de charge élaient supposés constants ou à peu près. Celte hypothise tait admissible puisque leurs calculs ont été confirmés par lexpérience.

Notre but est identique : déterminer la propa- gation de l'onde de marée résultant de modifieations de la gíométrie de l'estuaire. In calcul préliminaire nous a montré que les coefficients de perte de charge ne conservaient pas une valeur constante pendant la maré le calcul étail done impossible sans connaitre leur variation le long de l'estuaire au cours du temps. La connaissance de ces coefficients devrait nous permettre ágalement de procider plus facilement atu reglage des lignes deau of des courbes de maries de ce mene estuaire dont l'étude est efrectuie sur modile reduit.

Le volume des calculs a effectuer ne per- 
methail pas d'obtenir des résullats dans un délai compatible avec le programme d'élude sur modele roduil par les procédés classiques de calcul numérique. Aussi avons-nous utilisé les possibililes des nouvelles machines aulomatiques.
Nous allons présenter dans la suite quelques indications tres sommaires uniquement sur le calcul des pertes d'énergie (principe, exécution, résultats), cet exposé n'élant qu'un résumé du rapport complet sur le calcul.

\section{II. - PRINCIPE DU CALCUL}

\section{$1^{\circ}$ RAPpel DES ÉQuations :}

Les équations aux dérivées partielles (établies dins le cas d'un canal de pente et de profil constanls) régissant la propagation d'une intumescence à laquelle nous assimilons l'onde de maréc, sont les suivantes:

a) Equalion de continuité :

$\frac{\partial \mathrm{Q}}{\partial \boldsymbol{x}}+b \frac{\partial \mathrm{H}}{\partial t}=0$

(1) dimension $\mathrm{L}^{2} \mathrm{~T}-1$

b) Equation de la dynamique :

$$
\frac{\partial}{\partial x}\left(y \mathrm{H}+\frac{1}{2} u^{2}\right)+\frac{\partial \mathrm{U}}{\partial t}+g i_{r}=0
$$

(2) dimension $\mathrm{I}, \mathrm{T}-2$ où les notations sont classiques et où :

$\zeta=$ vilesse moyenne spatiale dans la section $S$,

$$
\mathrm{S} . \mathrm{U}=\iint u \cdot d \mathrm{~S}
$$

(a vitesse au centre de l'ément $d \mathrm{~S}$ ).

$i_{r}==$ pente due a la resistance, à la turbulence et, d'une facon ginerale, à lous les phénomenes qui peuvent entrainer des ecarts par rapport aux equalions theoriques :

$$
\left|g i_{r}\right|=\frac{i \mathrm{I}^{2}}{8 \mathrm{R}}=\frac{g^{2} \mathrm{~T}^{2}}{\mathrm{C}^{2} \mathrm{R}}=\mathrm{F}
$$

Dans ces equations, la pente de fond n'interrient pas.

G.-H. Kevular [2], lenant compte de la repartition verticale des vitesses, obtient la forme suivante de l'équation de la dynamique :

$$
\begin{aligned}
& \frac{\partial}{\partial x}\left(g \mathrm{H}+\approx \frac{\mathrm{U}^{2}}{2}\right)+\frac{\partial U}{\partial t} \\
& +(1-x) \frac{\mathrm{U}}{\mathrm{S}} \frac{\partial \mathrm{S}}{\partial t}+g i_{r}=0
\end{aligned}
$$

avec :

x coefficient de répartition verticale des vitesses défini par:

$$
x \mathrm{U}^{*} \mathrm{~S}=\iint_{\mathrm{S}} u^{2} \cdot d \mathrm{~S}
$$

L'établissement de ces équations impose certaines conditions classiques aux dórivées de $S, U$, à la pente du profil et à la courbure de l'onde.

Les coefficients \% ont été calculés le long de l'estuaire à partir des résultals d'une campagne de mesures de la Division des Essais Extérieurs du Service des Etudes ef Recherches Hydrauliques de l'Electricité de France, portant sur 4.000 mesures; $\alpha$ varie entre 1 el 1,1 , les valeurs les plus fortes se trouvant a laval de l'estuaire ou le terme $U / \mathrm{S} . \partial \mathrm{S} / \partial t$ est faible. 1 . $[12 / 2$ est loujours un terme correctif.)

Au total, nous pouvons, comple tenu de la precision des autres mesures, prendre $x=1$.

c) Equation de l'énergie :

M. Allakp indique [3] une combinaison des deux équations précédentes qui pernet de calculer les perles d'énergie globale. Nous n'avons pas appliqué sa méthode générale, car nous ne possédions que peu de relevés de courants. (Un calcul de cubature nous a foumi les vitesses moyennes suivant la direction moyenne de l'bcoulement.)

En multipliant l'équation de continuité par (g $\left.\mathrm{H}-1 / 2 \mathrm{U}^{2}\right)$ et l'équation de la dynamique par $P Q$, et en effechant la somme, on obtient :

$$
\begin{aligned}
& \frac{\partial}{\partial x}\left[\mathrm{Q}\left(y \mathrm{H}+\frac{1}{2} \mathrm{U}^{2}\right)\right]+o b\left(g \mathrm{H}+\frac{1}{2} \mathrm{U}^{2}\right) \frac{\partial \mathrm{H}}{\partial t} \\
& 1 \\
& 2 \\
& +Q \frac{\partial U}{\partial t}+p Q g i_{r}=0
\end{aligned}
$$

chaque terme a la dimension $\mathrm{M} \mathrm{L} \mathrm{T}^{-3}$ de la dérivée d'une énergie par rapport à $t$ et à $x$, ce qui conduit à intégrer par rapport à $x$ pour oblenir des puissances, puis par rapport à $t$ pour oblenir des énergies. 
1. L'intégration par rapport à $x$ entre $x_{0}$ et $x_{1}$ des termes 1, 2, 3, 4 fournit des termes $1^{\prime}, 2^{\prime}, 3^{\prime}, 4^{\prime}$ qui ont la dimension $M L^{2}{ }^{r}{ }^{-3}$ d'une puissance et représentent :

1 ' la différence des puissances transmises à travers les sections $x_{0}$ et $x_{1}$;

2 ' la variation d'énergie potentielle de la masse d'eau comprise entre les sections $x_{0}$ ef $x_{1}$ pendant l'unilé de temps;

$3^{\prime}$ la variation d'énergie cinétique correspondante;

4' la puissance dissipée (par frottement, turbulence):

$$
4^{\prime}=P(t)
$$

2. L'intégration entre $t_{0}$ et $t_{1}$ des termes $1^{\prime}, 2^{\prime}, 3^{\prime}, 4^{\prime}$ fournit des termes $1^{\prime \prime}, 2^{\prime \prime}, 3^{\prime \prime}, 4^{\prime \prime}$ qui ont la dimension $\mathrm{M} \mathrm{L}^{2} \mathrm{~T}^{-2}$ d'un travail et représentent les analogues des quantités $1^{\prime}, 2^{\prime}, 3^{\prime}, 4^{\prime}$ pour l'énergie entre les instants $t_{0}$ et $t_{1}$.

En particulier :

- 1) Si $t_{1}=t_{0}+\mathrm{T}$, T période de la maréc, les différents termes représentent :

1 "la différence des puissances transmises a travers les seetions $x_{0}$ et $x_{1}$ pendant une marée;

2" la variation d'énergie potentielle, nulle si l'on admet qu'au bout d'une période, les conditions de forme et de vitesse de l'onde de marée sont identiques;

3" la variation d'énergie cinétique, nulle pour les mêmes raisons;

4 " le travail dépensé par frottement pendant une marée.

- 2) Si l'on inlègre entre étales successives, lo calcul permet de sćparer, énergies dissipées au llot et au jusant.

$P(t)$ représente la puissance dissipée sur l'aire A comprise entre les sections d'abscisses $x_{0}$ et $x_{1}$, soit $\mathrm{P}(t) / \mathrm{A}$ par unité de surface. En admettant que cetle puissance dissipée provienne uniquement du frottement et que la force de frottement moyenne par une unité de surface, $F$, soit proportionnelle à $\&\left(\mathrm{U}^{2} / 2\right)$ (écoulement turbulent), la puissance dissipée serait égale à :

$$
\mathrm{F} \cdot \mathrm{U}=\mathrm{C}_{f} \cdot \frac{\mathrm{U}^{*}}{2}
$$

d'où :

$\frac{P(t)}{\mathrm{A}}=\mathrm{C}_{f} \circ \frac{\mathrm{U}^{3}}{2} ; \frac{P(t)}{\mathrm{A} \cdot \mathrm{U}^{3}}=\frac{\mathrm{C}_{f}}{2}=\frac{\lambda}{8}\left(\lambda=4 \mathrm{C}_{\mathrm{f}}\right)$

d'où un procédé de calcul du coefficient universel de perte de charge.

\section{2* Principle de Cahcul \\ ET TRANSFORMATION DIS EQVATIONS.}

Le but du caleul est de determiner, à partir des lignes d'eau el de la géométric du bassin, les valeurs de $g i_{r}$ (d'où $\lambda$ ou $C$ ) et de $P(t)$;

- L'équation de continuilé fournit le débit Q el la vitesse moyenne $\mathrm{U}$;

- L'équation de la dynamique fournit $g i_{r}$ (d'où is et $\mathrm{C}$ ).

- L'équation de l'énergie fournit P $(t)$ (d'où $\lambda$ ).

Les équations aux dérivées particlles sont résolues numériquement en remplaçant les dérivées par des différences finies $\Delta / \Delta t, \Delta / \Delta x$ qui se calculent en considérant des aceroissements des fonctions a $x$ ou $t$ constant (suivant des droites parallèles aux axes des $t$ ou des $x$ ); les différences finies représentent des valeurs moyennes des dérivées sur un intervalle $\Delta x$ ou $\Delta l$. Il faut donc envisager, non pas la valeur des quantités $\mathrm{U}, \mathrm{H}$, $b$, $\mathrm{Q}$ en un point donné, mais la valeur moyenne dans un intervalle $\Delta x$ ou $\Delta t$ ou les deux suivant les cas.

Si l'on considère dans le plan $x$, $t$, le quadrillage formé par les droites de coordonnées respectives, $\Delta x_{1}, \Delta x_{1}+\Delta x_{2},+\ldots \Delta t, 2 \Delta t, 3 \Delta t, \ldots$, les valeurs des différentes variables $\mathrm{H}, \mathrm{S}, b \ldots$

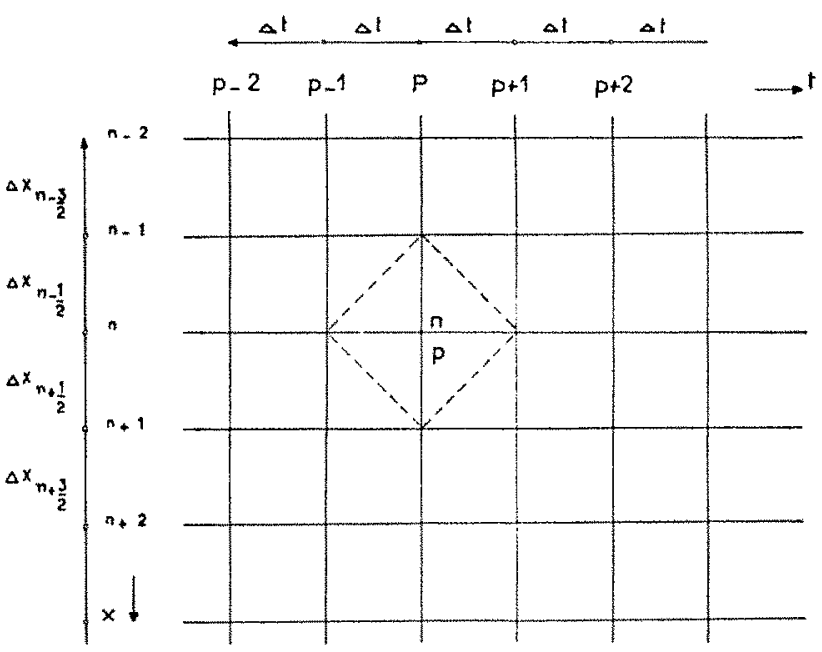

Fiti. 1. - Résean d'intégration.

sont connues, par exemple, aux nouds des quadrillages. Le calcul consiste à déterminer les inconnues au centre d'un reclangle, par exemple, en fonction des données aux quatre sommets correspondants, ou en un noud en fonction des données aux sommets du losange dont le noud est le centre (fig. 1). 


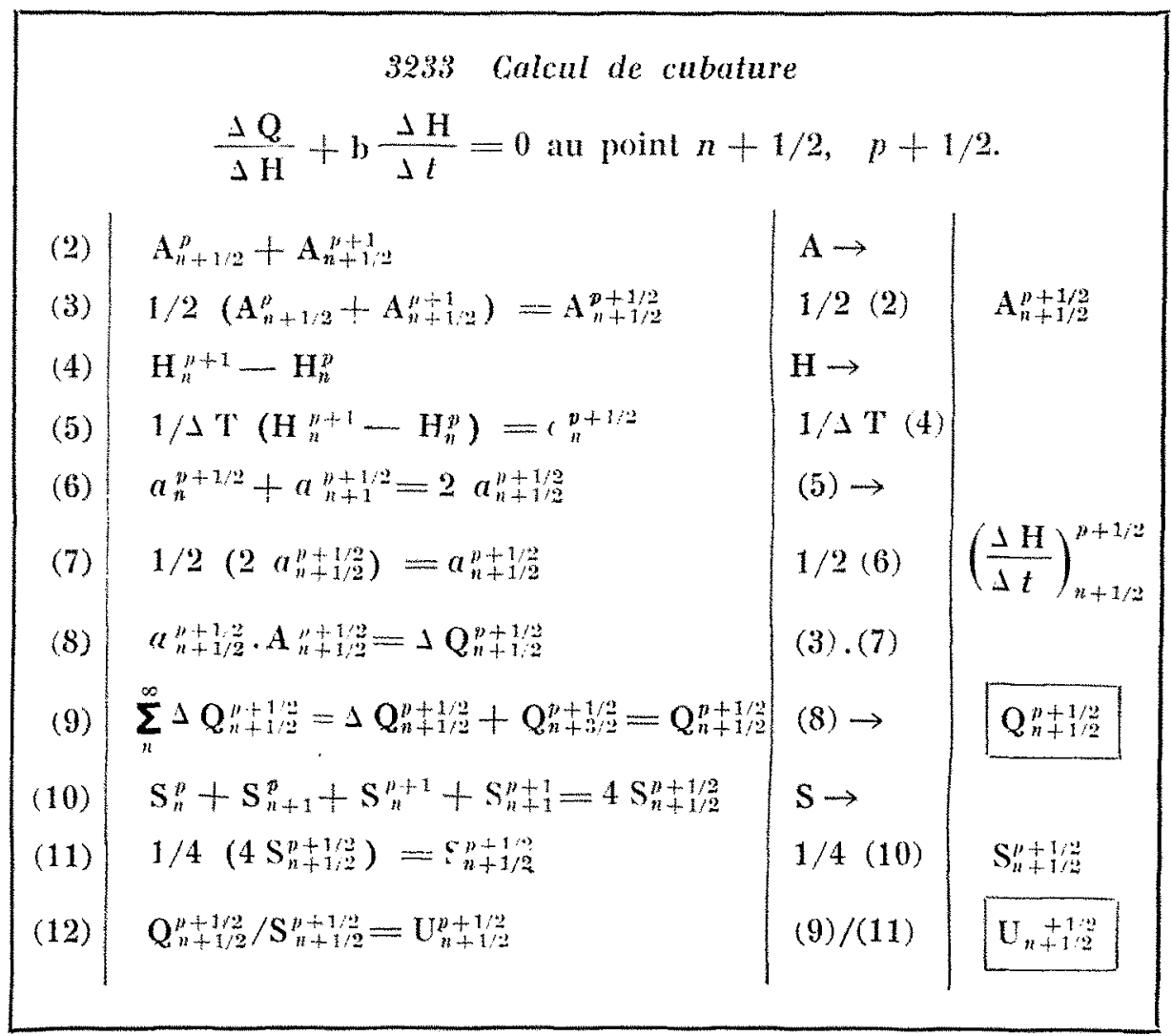

F16. 2. -- Planification du calcul de cubature.

Les ealculs de cubature et de coefficient de perte de charge ont été effectués au centre d'un rectangle (d'où valeur moyenne dans un troncon).

Le calcul effectué en un noud fournit des résultats dans une section déterminée (calcul de l'énergie qui traverse une section déterminée).

\section{3" Phanification du chleul}

(Exemple: cubature fig. 2).

Lat planification du calcul consiste à décomposer la suite des opérations en opérations élémentaires que la machine est capable d'effectuer (addition, soustraction, multiplication, division, extraction de racines, sommation, $\sin x / x$, $\mathrm{s} h x / x, \cos x / x$, ch $\left.x, e^{x}\right)$.

Connaissant les valeurs de $b, H, S, u, A$ en fonction de $x$ et $t$, les trois calculs se décomposent en 90 opérations élémentaires.

Une difficulté s'est présentée au confluent de deux rivières car le calcul dans un troncon nécessite la connaissance de résultats dans le tronçon amont, nous avons été amenès à considérer une section fictive formée par la réunion des dcux sections adjacentes à l'embouchure et à calculer dans cette section des valeurs fietives de certains résultats. L'introduction des conditions aux limites a pu s'effectuer simplement.

\section{1. - EXECUTION DU TRAVAIL}

Les calculs ont été exécutés au Bureau de Calcul numérique de la Cie I.B.M.; les indications qui suivent sont tris sommaires, car nous ne pouvons entrer, ici, dans le détail du fonctionnement et de la codification.

\section{Groupe calculateur I.B.M. (cl organigramme fig. 3).}

Le groupe calculateur électronique I.B.M. est une machine arithmétique où le programme est 


\section{ORGANIGRAMME DE FONCTIONNEMENT \\ DU GROUPE CALCULATEUR ÉLECTRONIQUE IBM}

(à programme par corles pertorées)

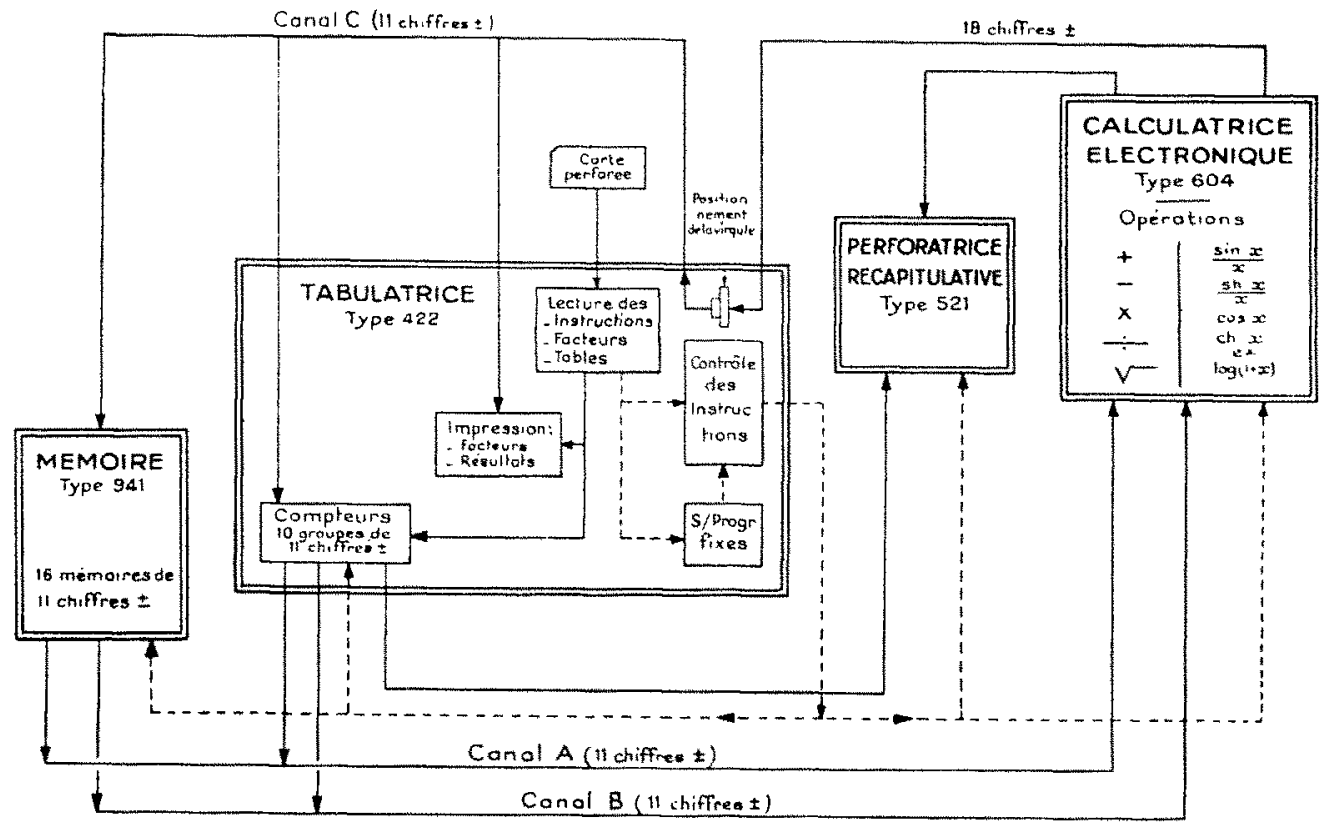

FIG. 3

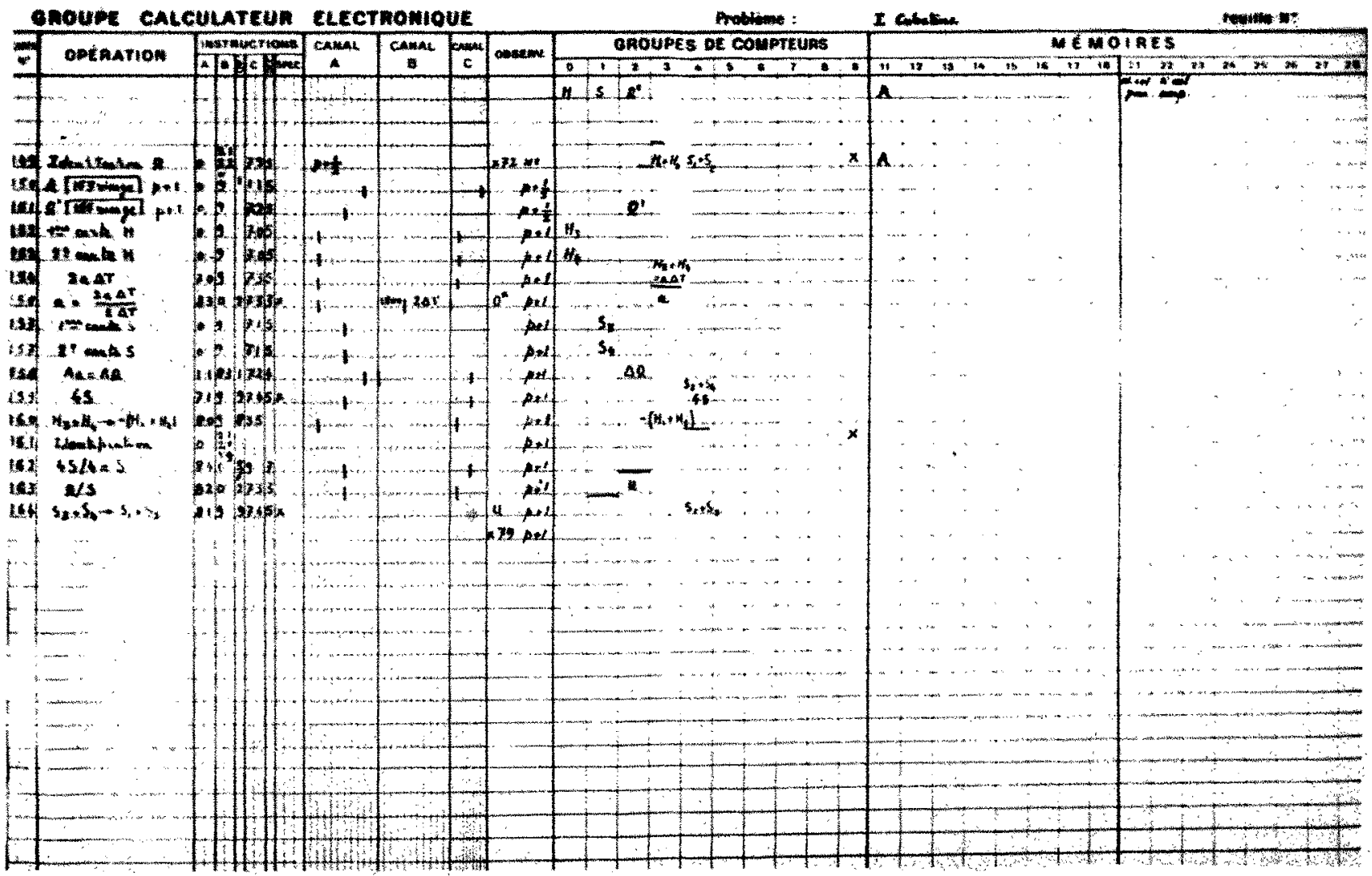

Fic. 4. - Calcul de cubature. Codification. 
introduit par des cartes perforées. Il comprend quatre ćléments : tabulatrice, mémoire, calculatrice électronique, perforatrice.

- La tabulatrice a pour rôle de lire sur les cartes perforées les instructions du calcul et de les transmettre aux différents organes du groupe. Elle peut imprimer des résultats.

- L'unité de mémoire permet de conserver de façon semi-permanente des résultats intermédiaires, des constantes.

- La calculatrice effectuc, sous le contrôle de chaque carte instruction, les différentes étapes du programme, codifices sur la carte et les opérations élémentaires que nous avons énoncées.

- La perforatrice récapitulatrice permet de perforer les résultats sur cartes.

\section{Cartes perforées.}

Les données sont introduites sous forme de cartes perforées et chaque opération est codifiée au moyen d'un code à trois adresses (adresse des deux facteurs de l'opération à efrectuer el adresse du résultat).

\section{$3^{\circ}$ Copificatron}

(Exemple : calcul de cubature, fig. 4).

La codification consiste à traduire en chiffres conventionnels de la machine tout le détail des opérations indiquées dans la planification.

\section{Temps D'exECUTION.}

Dans un calcul de ce genre, le travail de planification et de codification est de beaucoup le plus long.

Nous avons effectné deux calculs, le premier en prenant $\lambda t=15 \mathrm{~m}$, le deuxieme en prenant $\Delta t=30 \mathrm{~m}$. Nous avons choisi 20 sections dans le domaine fluvio-maritime.

Le calcul complet pour un tronçon et pour un intervalle déterminés comporte 90 opérations élémentaires :

- Dans le premier calcul, $\Delta t=15 \mathrm{~m}$, le nombre d'intervalles $\Delta t$ est de 50 , d'où au total :

$20 \times 50 \times 90=90.000$ opérations.
- Dans le deuxième calcul $\Delta t=30 \mathrm{~m}$, nous avions 45.000 opérations.

- Les données introduites sont : lirants d'eau $\mathrm{H}_{n}$, sections mouillées $\mathrm{S}_{n}$, largeurs en surface $b_{n}$, surfaces des plans d'eau $A_{n}(20 \times 50 \times 4=4.000$ données $)$, plus longueur des troncons, les cotes des zéros des marégraphes $(2 \times 20$ données) et des constantes S, T, $\gamma, \odot$, soit un peu plus de 4.000 données.

Pour déterminer ces valeurs, nous avons tracé leurs variations en fonction du tirant d'eau, puis, connaissant la courbe de marée $h(x, t)$, nous en avons déduit les valeurs des données en fonction de $x$ et de $t$.

Dans chaque tronçon et pour chaque intervalle, nous avons relenu 14 résultats, soit : 14.000 et 7.000 résultats respeclivement dans le premier et le deuxième calcul.

\section{5" Avantages, inconvénients.}

\section{a) Avantages:}

- Rapidité. Les travaux préliminaires de détermination des donnes (à effectuer quel que soit le mode de calcul) et de codification étant effectués, le calcul nécessite quelques dizaines d'heures de fonctionnement de la machine. Un travail analogue pour des intervalles plus larges avait nécessité trois mois de travail, avee une machine à calculer ordinaire à clavier.

- Précision. Les résultats sont fournis avec le nombre de décimales désirées. La machine étant une machine arithmétique, la précision des résultats ne dépend que de la précision des données des équations, du mode de caleul et du découpage en $x$ et $t$.

b) Inconvénients (ils sont, en général, mineurs) :

- Erreurs. Dans les machines analogiques, l'erreur principale est l'erreur de lecture : dans les machines électroniques, l'erreur vient des données ou est puremenl accidentelle. Des erreurs systématiques peuvent s'introduire lorsçu'on modifie accidentellement l'ordre des cartes perforées.

- Dépouillement. Les résultats sont présentés sous forme de tablean de chiffres. Dans les machines analogiques, on obtient directement des courbes de variations. 
IV. - RESULTATS

Le calcul dont nous présentons quelques résultats a été effectué pour le domaine fluviomaritime de la Gironde dans le cas d'une marée de coefficient 108 et de débits lluviaux amont moyens. Les résultats que nous avons oblenus sont au nombre de 14 pour chaque tronçon et chaque intervalle de temps considéré. Ce sont :

- Calcul de cubature : débit Q el vitesse moyenne $\mathrm{U}$

- Calcul de coefficient de perte de charge.

- accélération due a la pente de la ligne d'eau $g \frac{\partial \mathrm{H}}{\partial x}$

- accélération due à la variation du carré de la vilesse $\frac{1}{2} \frac{\partial U^{2}}{\partial x}$

- accélération due à la variation de vitesse : $\frac{\partial \mathbf{U}}{\partial t}$
- accélólion fue au frollement $F$ (d'où el $C$ )

(b) $(7)(8)$

- nombre de RExions d

- Calcul d'énergie.

- énergie traversant une section

$\mathrm{Q}\left(g \mathrm{H}+\frac{1}{2} \mathrm{U}^{2}\right)=\mathrm{Y}$

- énergie potentielle

$\int_{s_{1}}^{r_{2}} \mathrm{Q} \frac{\partial \mathrm{U}}{\partial t} d x=\mathrm{l}$

- energie cinétique

$? \int_{w_{1}}^{x_{2}} b\left(g \mathrm{H}+\frac{1}{2} \mathrm{U}^{2}\right) \frac{\partial \mathrm{H}}{\partial t} d x=13$

- puissance dissipée $P(t)$

- coefficient universel de perte de elzarge $\lambda$

1" Galcul de cubature (fig. 5 a 6 ).

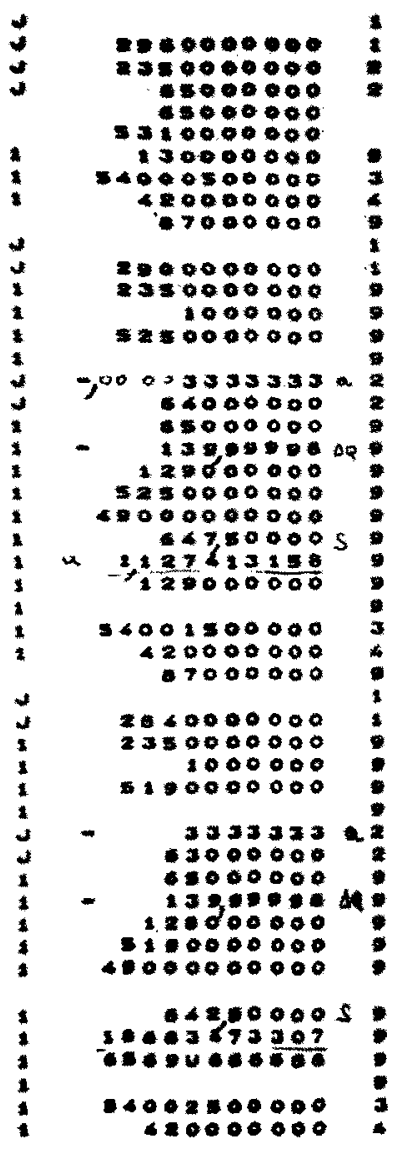

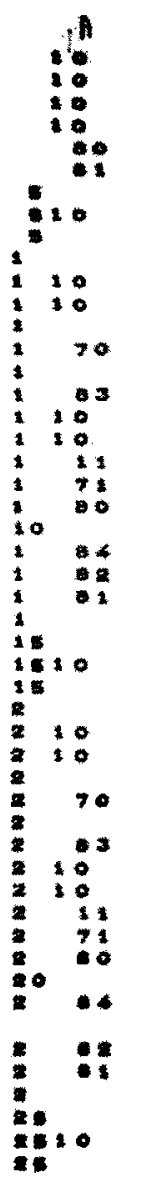

(2), $5=0000000$ 4010000 31000000 37100.0000 $16000 \% 00000$ 200,00000 10,00200

2,000000000 3,30000000 5,200000000

0,00000000 460,00000 120000000 12000000 1500000 $1,2 \pm 000000$ $=000000$ $2 \$ 0,0000$ 120,00000

34001300000 420000000 150,00000 200000000

100000000

0,100000000 3000000 1300000 200000 1200,00000

13000000

1000000000

15000000

septityeo

- $400=00000$ \%0:00\%००

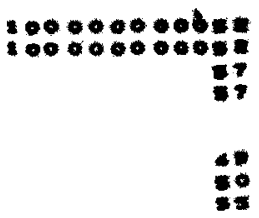

10000000005 100000000002

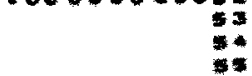

00,000000086

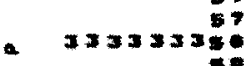
10 $00000 \%$ 3

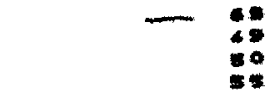

$1000000000 \%$ 100000000082

3 10000000000

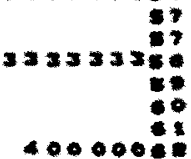

:

Fig. 5. - Caleul de cubature. présentation brute des résultats. 


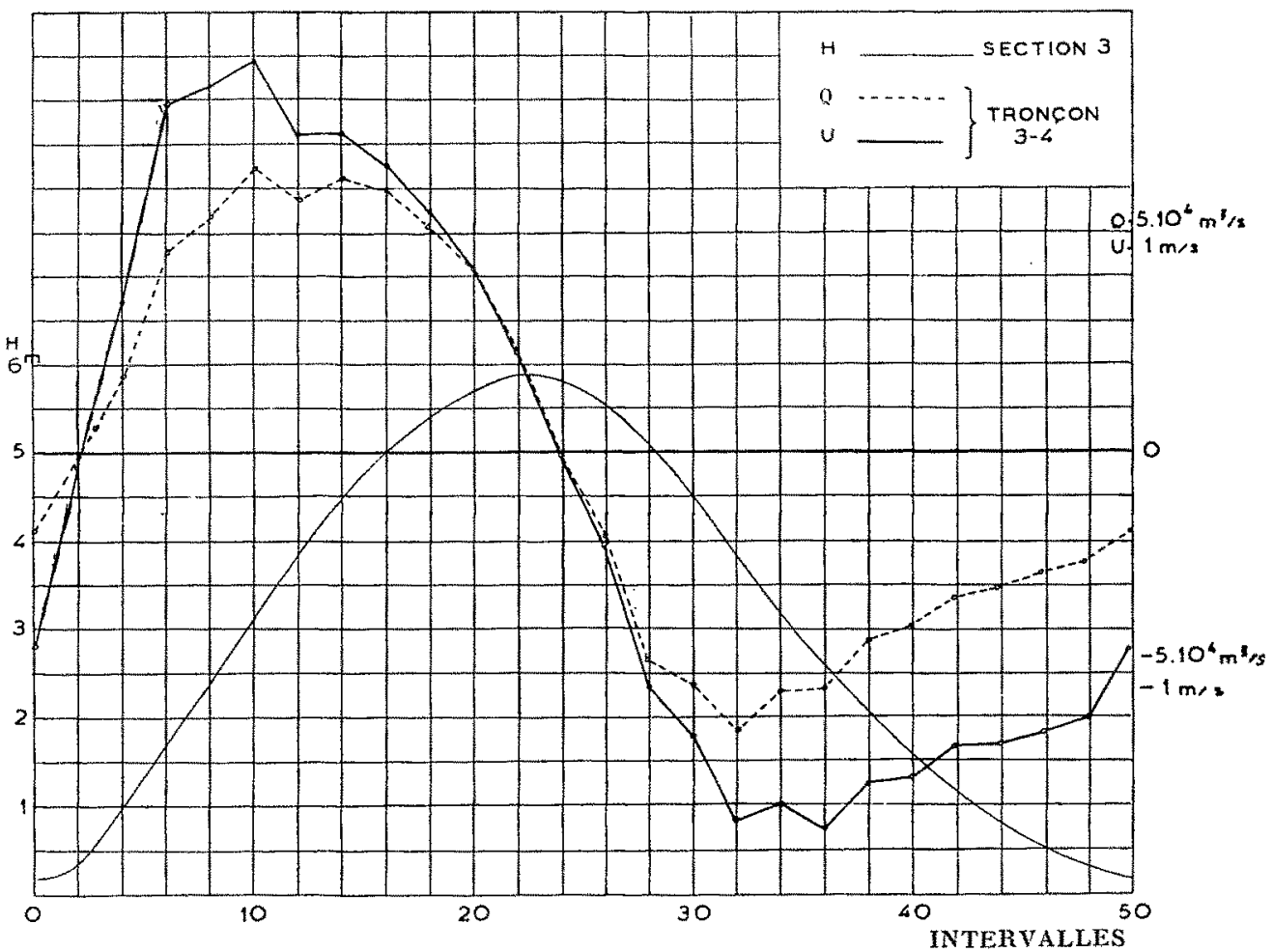

F19. 6. - Calcul de cubature.

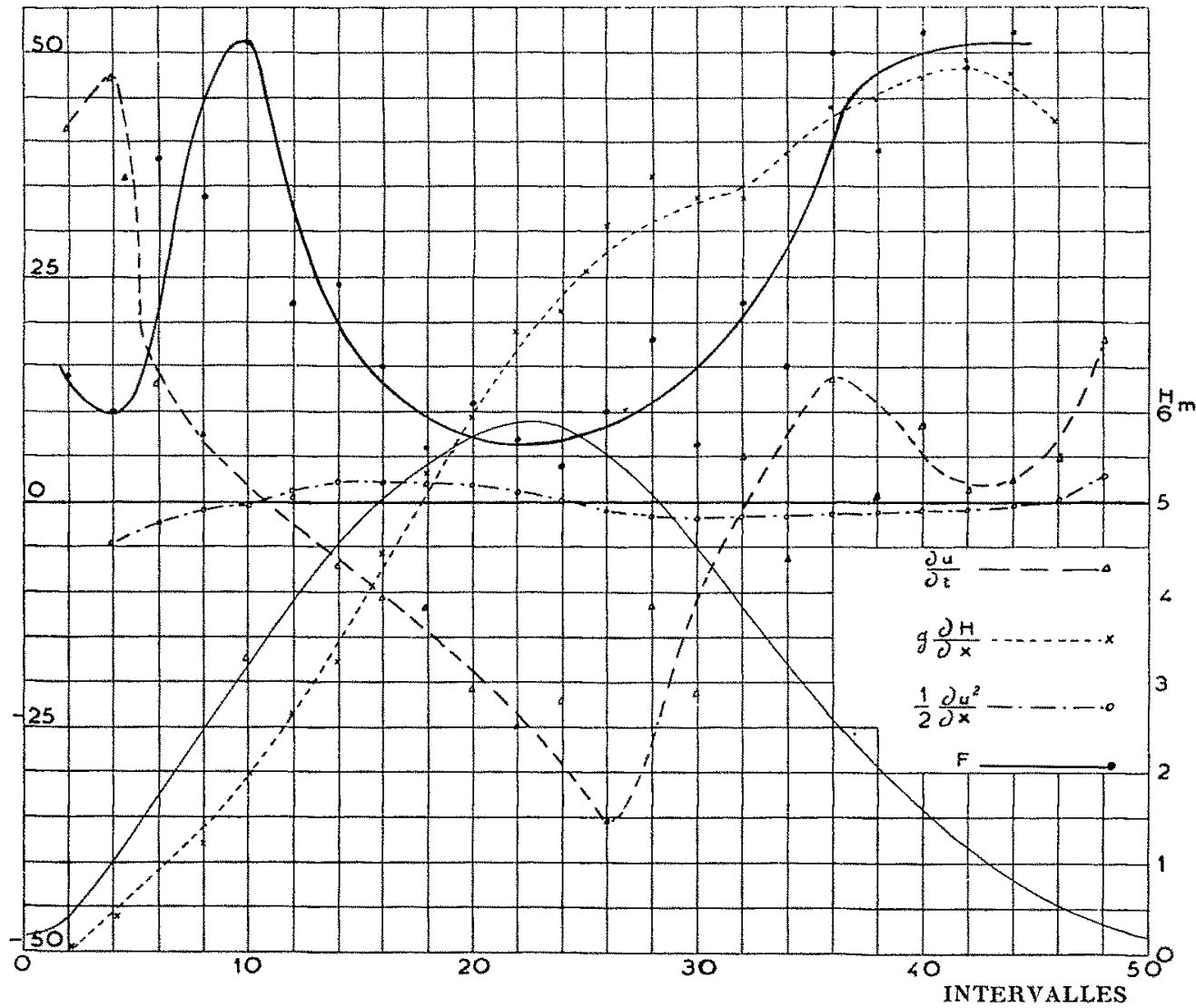

Fis. 7. - Calcul de la perte de charge (3-4). 
Ce calcul, le plus simple, n'entraine aucune remarque particulière. Nous avons obtenu des valcurs semblables aux valcurs calculées par d'autres procédés. A remarquer que les renverses de courants sont légèrement postérieures aux étales de niveau.

$2^{n}$ Calcul des coefficients de perte de charge.

a) Analyse des différents termes (fig. 7) (pente en $10^{-5}$ ) :

- $g \frac{\partial h}{\partial x}$ ce terme est proportionnel à la pente de la ligne d'eau dans la section considérée (au flot $<0$, au jusant $>0$ ).

- $\frac{\partial U}{\partial t}$ ce terme représente l'accélération longitudinale des particules d'eau. Ses valeurs ne sont pas très continues, car son calcul fait intervenir les valeurs des vitesses dans les deux intervalles adjacents.

$-\frac{1}{2} \frac{\partial^{2}}{\partial x}$ ce terme est faible comparé aux deux autres.

- F représente la valeur absolue de la somme de ees trois termes. b) Valeur du coefficient de perte de charge:

Les courbes représentatives présentent quelques anomalies, surtout au moment des etales de courant. En effet, à ces instants, les hypothèses d'établissement des équations ne sont plus vérifiées (fig. 8).

1. La figure 8 fournit les variations de $\lambda$ en fonction de $t$ dans les tronçons $3-4,7-8,12-13$, $17-18$ avec comme valeur moyenne 0,009 dans $3-4,0,026$ dans 7-8. L'allure de la variation est nettement différente au flot (fortes varialions) et au jusant (valeurs à peu près constantes).

A l'amont, les tronçons 12-13, 13-14 fournissent respectivement $\lambda=0,025$ et $\lambda=0,017$.

2. La figure 9 fournit les valeurs de $\lambda$ sur l'ensemble du domaine fluvio-maritime et permet les remarques suivantes :

- troncon $0-1 \quad \lambda_{m}=0,020$

forte perte de charge due it l'élranglement;

- troncon 5-6 $\lambda_{m}=0,012$ présence d'île;

- tronçon 6-7 $\lambda_{m}=0,012$

-.- tronçon 7-8 $\lambda_{m}=0,026$

présence d'ile - grande difficulli dans le réglage des lignes d'eau.

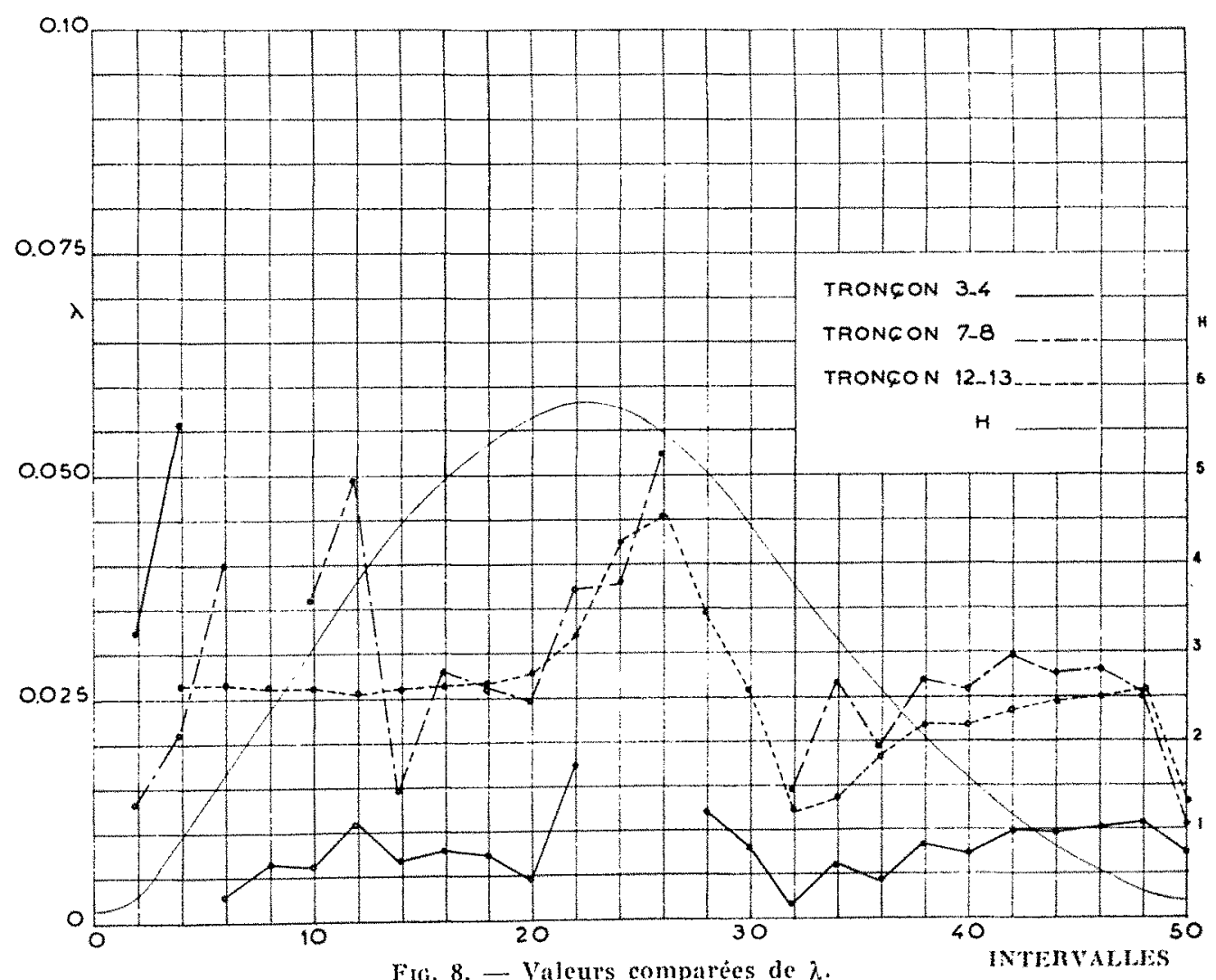




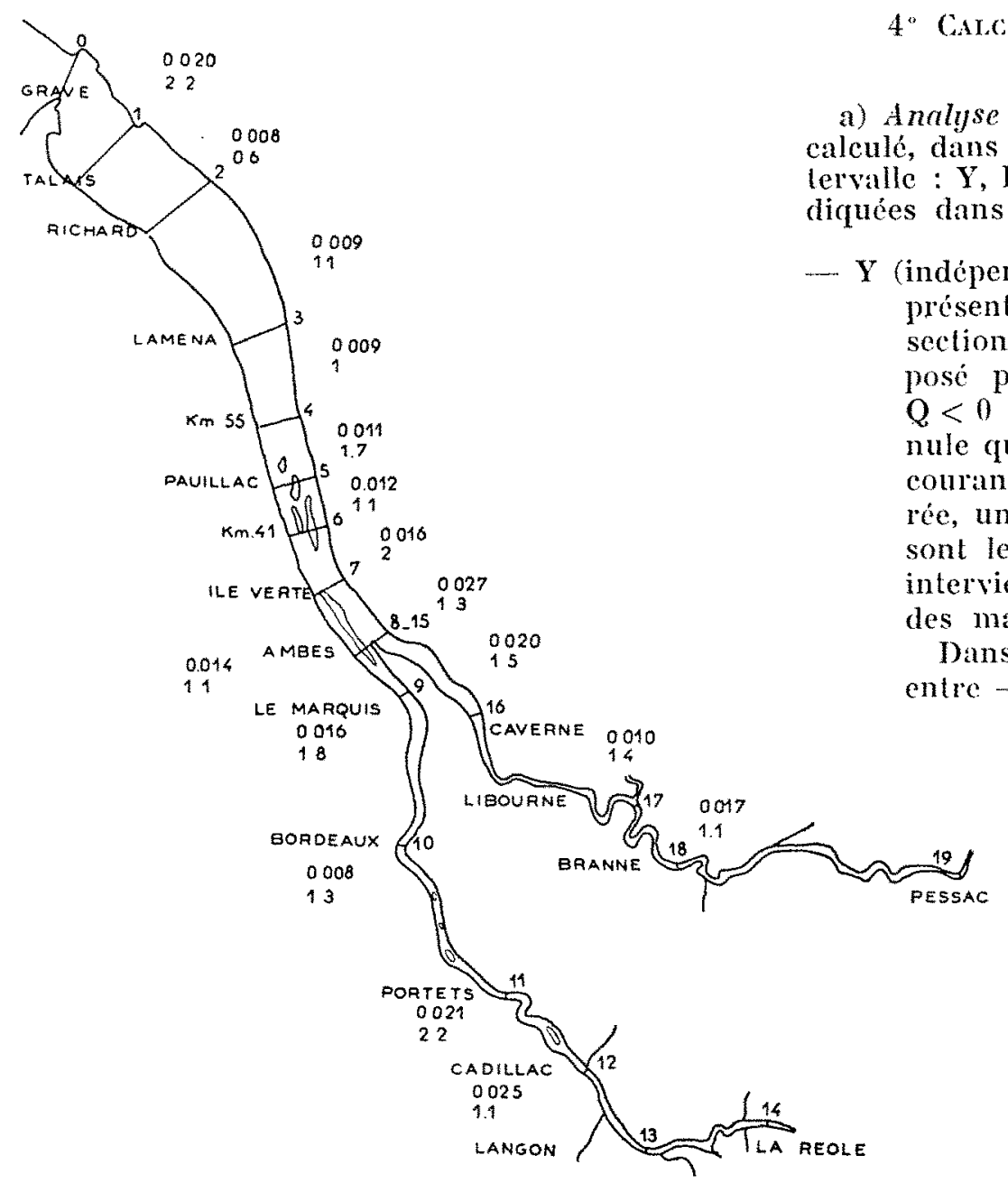

Fici, 9. - Valeurs du coefficient $\lambda$ et de la puissance dissipée par $\mathrm{km} 2$.

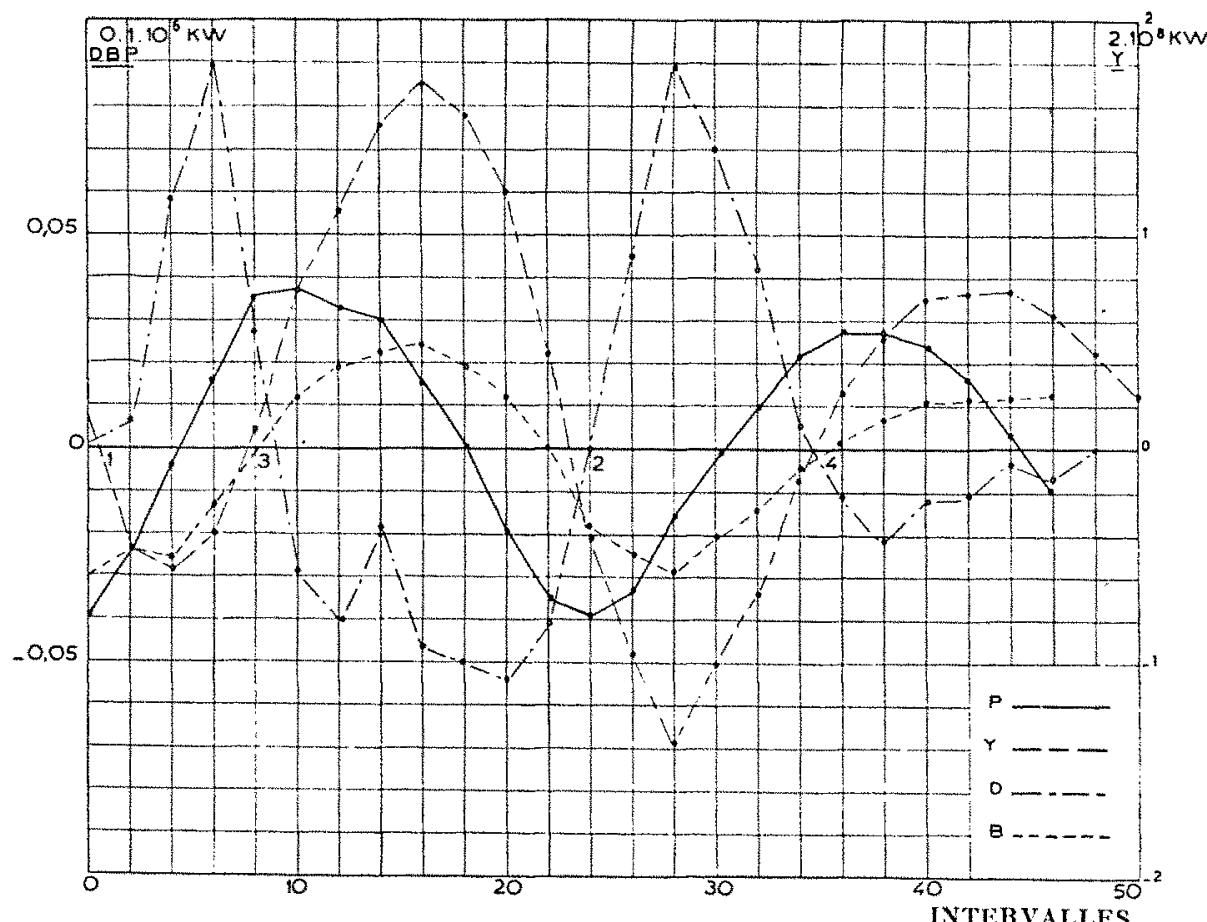

Fra. 10. - Calcul de perte d'énergic (3-4). 
- D représente les variations de $\int Q \frac{\partial U}{\partial t} d x$ (énergie cinétique). Dans le troncon 3-4, $D$ varie entre - 0,05 et 0,09 mégakilowatt. L'intégrale de D sur la marée doit être théoriquement nulle. Le calcul fournit une valeur moyenne de 1,4 mégawatt, soit $2 \%$ de la valeur maxima de $\mathrm{D}$ pendant la marée.

- B représente des variations de :

$$
\bullet \int b\left(g \mathbf{H}+\frac{1}{2} \mathbf{U}^{2}\right) \frac{\partial \mathbf{H}}{\partial t} d x
$$

(énergie potentielle), indépendant du niveau de référence. Sur la marée complète, l'intégrale doit être nulle. Le plan de référence choisi étant le plan $O$ du N.G.F. Dans le tronçon 3-4, B varie entre $-0,25$ et $+0,25$ mégakilowatt. L'intégrale sur la marée est de -9 mégawatts, soit $3,5 \%$ de la valeur maximum de $B$.

- $P$ représente la puissance dissipée à chaque instant par les forces de frottement et par les autres causes de perte de charge telles que : rétrécissement, élargissement..., par suite de la substitution à l'estuaire d'une série de tronçons rectangulaires dont la section est égale à la section moyenne du tronçon réel. Tous les écarts par rapport au mouvement théorique sont pris en compte par le terme de frotlement; $P$ est donc supérieure à la puissance dissipée par frottement pur. A partir de la valeur moyenne de $P(t)$, nous avons calculé la valeur de $\lambda$ moyen que nous arons trouvée égale dans 3-4 à 0,009 (dans le premier calcul $\lambda=0,009$ ) Les valeurs instantanées de $P(t)$ nous ont permis le calcul des valeurs instantances de $\lambda$; leur valeur moyemne dans $3-4$ est 0,008 (au lieu de 0,009 par les deux autres procédés de calculs).

b) Résultats (fig. 9) :

1. Nous avons exprime $P$ en $10^{\circ} \mathrm{kW}$ par $\mathrm{km}^{2}$. La puissance dissipée est de l'ordre de 1 à 2 mégawatts par $\mathrm{km}^{2}$.

La puissance dissipée varie entre $0,6 \mathrm{ct}$ 1,5 mégawatt par $\mathrm{km}^{2}$ a l'aval de l'es- tuaire (sauf à l'embouchure où elle est de 2,2 mégawatts par km². Liembouchure présente, d'ailleurs, un rétrécissement tres important).

Dans les zones très perlurbées par la présence d'ìle, la puissance dissipéc varie entre 1,5 et 2,2 mégawalls par $\mathrm{km}^{2}$. Les zones à forte valeur de $P / A$ correspondent aux zones où, sur le modèle, nous avons été obligés de créer les pertes de charge très concentres pour obtenir les lignes d'eau convenables.

2. Séparation des pertes d'énergie all flot el an jusant: Le calcul ne peut pas s'effeeluci directement par la machine, car elle ne peut ehoisir les instants des étales de courant. Nous avons effectué ce ealeul pour quelques sections, celles où la dissipation d'énergie est importante.

Les pertes d'énergie et les coefficients universels de perte de charge sont, en gónéral, plus importants au jusant qu'atu flot géometrique de l'estuaire qui, par rapport à l'écoulement, est un convergent au flot et un divergent au jusant), sauf dans la section aval qui présente la disposition inverse.

Dans le troncon 6-7 ( $\mathrm{km} 41$, lle Verte), le calcul fournit pour $\lambda, 0,010$ au fot, 0,020 au jusant, 0,016 sur toute la marée.

3) A partir des valeurs calculées de $\lambda$, nous pouvons determiner la propagalion de l'onde de mare par diverses mithodes : Holsters, Cray, Scholnfeld, L'application de la méthode Scholsmes nous a permis de déterminer le point $(x, t)$ ou apparaissait le mascaret en Garonne.

Nous avons également envisagé dutiliser les machines électroniques pour ce ealcul. Comple tenu des possibilités actuelles du groupe caleulateur I.B.M. (qui est une machine arithmétique), nous n'avons pu déterminer un processus comm mode, la machine étant incapable d'effectuer, du moins de façon simple, les choix successifs qui s’imposent dans la suite du tracé des caractérisliques. Il nous semble que, dans celle voie, on doive s'orienter vers les machines analogiques qui ont déjà effectué des calculs d'oscillation en masse et de coup de bélier.

\section{V. - UTILISATION DES MACHINES ARITHMETIQUES}

Les calculs précédents n'ont pu être effectués que grâce à la rapidité des nouvelles machines mathématiques dont les possibilités modifient les théories d'analyse numérique : l'exemple le plus simple est fourni par les calculs de quadrature. La méthode de calcul démentaire des trapizes 
a été perfectionnée de façon à diminuer les nombres des opérations (Gavss, Wreble...). Avec les machines mathématiques, le procédé le plus simple consiste à appliquer brutalement la méthode des trapezes; toutes les valeurs successives des cotés des trapèzes sont envoyées dans un compteur qui fournit ainsi le résultat.

Les machines mathématiques sonl de deux types : arithmétique et analogique; les machines arithmétiques (groupe calculateur I.B.M.) opèrent sur des quantités discrètes. Les machines analogiques opèrent, au contraire, sur des quantités physiques pouvant varier de facon continue.

Les machines mathématiques peuvent résoudre les problèmes suivants: systìmes d'équations linéaires (jusqu'à 20 inconnues), équations différentielles et systèmes d'èquations différentielles, équations aux dérivées particlles (difficultés pour l'introduction des conditions aux limites). Les machines sont donc particulierement bien adaptées aux problèmes linéaires (vibrations, élasticité, matrices, déterminants). Elles sont la base de tout calcul statistique.

Dans la littérature, on s'aperçoit que les applications ont surtout été développées dans des domaines liés aux industries de guerre en particulier : balistique (tables de tir américanes), mécanique (torsion), physique nucléaire (équation de Schröprogra) et dans les calculs de table de fonctions.

En mécanique des fluides, les applications principales ont ité faites en aérodynamique qui a bénéficié, pendant la guerre, de l'essor de l'aviation. En hydrodynamique, les applications sont plus restreintes et pourtant de nombreuses études conduisent à des équations inintégrables dans la plupart des eas, mais dont les solutions pourraient être facilement tabulées. Ainsi, dans une communication sur le probleme des « rollwaves $», M$. Dressuer fournit une solution dépendant de fonctions dont la tabulation permettrait de confronter théorie et expérience.

Dans le domine de l'hydraulique, on essaye de ne pas recourir aux calculs numériques, car les procédés classiques sont toujours longs et fastidieux. Les machines mathématiques permetlent de pallier ces inconvénients. Un calcul numérique, même très complexe, n'est plus un obstacle. La possession d'un outil aussi puissant que les machines mathématiques modernes permet d'envisager les problèmes sous un angle absoItment nouveau.

\section{BIBLIOGRAPHIE}

(1) Dronkras J.J. -- De Ingenien, 5-10-51, n" 40, 137.146. Calcul de marées.

[2] Kertegan G. H. - Resentch Paper, R P 154t. National Bureau of Stmulares. Effects of lurbulence and channel slope on translation waves.

[3] Alfard P. - Annales de Geophysique, janvier-mars 1951 , pp. 9-44. Forme te energie de l'onde de maré de vive eau entre les Héaux de Brehat et le cap de la Hague.

\title{
DISCUSSION
}

\author{
(Président : $\mathrm{M}$, Hepser)
}

M. le Président remercle M. FatnE et souligne le grand intérèt à la fois theorique et pratique de sa communication.

M. Meyer signale que les Ets Neypie ont conslaté que les calculs qu'ils ont faits avee la machine a carte perforée comportaient des erreurs de l'ordre do 20 a $25 \%$, dues principalement au remplacement des dérives pas des differences finies; il demande a M. Fatre quelles sont les dispositions a prendre pour eviter ces creurs.

M. Fatre répond que les résultats obtenus au Laboratoire National d'Hydraulique a moyen de la machine et arec le caleul direct sont du mème ordre de grandeur : en particulier, pour les débils et les vilesses moyennes, les valeurs ealculées à partir des relevés de la répartition verticale des vitesses, effectués par la Division des Essais Extérieurs du Service E.R.H. d'E.D.F, ne présentenl aucune difference avec les valeurs donnes par la machine a carte perforéc.

M. Meyen dit qu'il s"est toutefois rendu compte que les resultats changent suivant que l'on fait la sommation des differences finies suivant laxe des $x$ (déplacements) ef l'axe des $t$ (temps), ou les directions de propagation des ondes. Ces dernieres semblent les meilleures; malheureusement dans fo cas des canaux il y a deux vitesses differentes pour les ondes ot il faut que la machine marche toujours plus vite que les ondes, autrement les résultats deviennent completement faux.

Une meilleure approximation serait obtenue en poussant jusqu'aux dérivées d'un ordre supéricur au second, vaisemblablement $y^{\prime *}$ on $y^{17}$ au licu de $y^{\prime \prime}$. Ceci reviendrait physiquement a tenir comple de 8 à 10 points au licu de 4 par rectangle.

M. Fache repond quo dans l'exemple cité, les vitesses qui sont introduites dans le calcul sont déterminées à partir de valeurs qui, pratiquement, font intervenir les quatre ordres de dérivation.

Sur la demande de M. le président, M. Meyen précise : 1" Que le Laboratoire Dauphinois d'Hydratique n'a pas encore appliqué les procédés de caleul mécanique à des modèles en cours d'études, mais a tenu d'abord a vérifier ses résulfats sur un cas sehematique constitué par un canal rectangulaire dans lequel a été réalisé un écoulement de caractéristiques connues; la mise a point de l'emploi de la machine sera poursuivic avant son application aux etudes cn cours.

$2^{n}$ Que les méthodes graphiques ne prêtent pas aux mèmes eritiques que celles qu'il signale dans le calcul par machine bien que les unes et les autres fassent intervenir des différences finies ear les méthodes graphiques integrent suivant les ondes, alors que la machine prow 
cide suivant les axes de coordonnés, ec qui introduit les erreurs signalées plus haut.

M. Fatre rappelle que le but du calcul qu'il a exposé consistait en la determination des pertes de charge el que les résultats obtenus sur ce point sont suffisamment préeis, les autres raleurs n'étant indiquées qu'acessoirement et comme ordres de grandeurs.

M. le Président remarque l'intérêt des observations de M. Meyen, dont la conclusion serait la nécessité de choisir judicieusement dans le plan du caleul le systeme de coordomnées et, éventuellement, la direction des axes.

II. Fatine précise que les erreurs ne sont pas dues á la machine elle-même, mais au procédé d'intégration.

Sur la demande de M. le Président, M. Fatine rappelle que pour le problème de la Gironde :

1 "Les données étaient les courbes de marée et les points de sondage; les vitesses n'étant connues qu'en un nombre restreint de points, l'équation de continuité a permis de déterminer l'ensemble des va+ leurs de cet élément dont on a vérifié la concordance avee les quelques valeurs connues (débits au bec d'Ambès notamment.

$2^{n}$ Les quantités d'ènergie dissipée ont èté trouvées égales à :

a) Pour la marée cludiée (coefficient 100) : $0,85 \mathrm{MW}^{\mathrm{T}}$ par $\mathrm{km}^{2}, 8,5$ millions de $\mathrm{kWh}$ pour l'ensemble de l'estuaire (surface $400 \mathrm{~km}^{2}$ );

b) Pendant une année comprenant 705 marées, en prenant le coefficient 0,5 pour ramener à la moyenne: 2,9 milliards de kWh.

(A titre de comparaison, M. Allatin a trouvé pour la Manche, entre la Bretagne et le Cotentin. soit pour $0.000 \mathrm{~km}^{2}$ de surface, 106 milliards de kWh par an.)

II. Allard fait les remarques suivantes :

1. La différence trouvée entre l'énergie dissipèe en Gironde d'après les calculs de M. Faune et celle qu'il a trouvée lui-même pour le golfe de Saint-Malo provient particulièrement du falt que l'unite de hateur dans cette partie de la Manche a une valeur approximativement double de celle qu'elle atteint en moxenne en Gironde. La nature des fonds a aussi une incidence : en Gironde le mourement liquide se produit dans un canal de section assez irrégulière dont une partie est entretenue artificiellement par des dragages. En Manche, il s'agit d'une région avec récif's, rochers, bancs de sable ct toutes les irrégularités des fonds naturels, done une région au relicf sousmarin très complexe. Il est par suite bien difficile d'interpréter a priori les différences entre les chiffres obtenus pour les énergies dissipées dans les deux domaines en cause.

2" En ce qui concerne les dornées relatives aux vitesses, il n'y a pas lieu de regretter que celles-ci ne solent pas données par l'expérience, par mesure directe; l'équation de continuité appliquée dans un canal permet de calculer la vitesse moyenne dans une section qui est précisément la quantité dont on a besoin et qui échapperait à une mesure directe faite en un point isolé. En pleine mer où les débits liquides ne sont pas canalisés. on est obligé d'utiliser des mesures directes de courant faites en un point ou plusicurs points, faute d'un renseibnement intégrè qui serait préférable.

$3^{*} \mathrm{Ce}$ qui, en tout clat de cause, reste délical dans co genre de calculs est l'éaluation des dérives quel que soit le procédé embloyé pour y parvenir : qu'il soit graphique ou mécanique tel qu L'arantage du procede graphique est d'amortir les resultats des mesures en en prenant la moyenne, le procede do calcul mécanique risque peut-être d'être plus brutal mais il presente incontestablement lavanlage de la rapidité.

Sur la demande de M. le Présidenl, MI. Fave précise que la seule erreur humaine dans l'emploi de la machine consiste en linversion des cartes, ee qui donne alors des resultats tout a fait incoherents.

M. Mryen estime toutefois qu'en général les machines a calculex peuvent introduire des erreurs inexplicables, sans doute par substitution d'un chifre a un autre au cours des opérations, ainsi gu'il a pa le constater récemment dans un très simple ealcul de puissance de groupe hydro-électrique.

M. FAvRe indique qu'arant le calcul d'intégration, tous les calculs intermediaires avaient été faits a la machine a calculer, vérifiés à la règle à calcul et, érentuellement, rectifiés.

M. le Président, pensant à la possibilite dappliguer cette méthode de calcul aux problèmes d'élasticité qui comportent de nombreuses équations aux dérivées partielles arec conditions aux limiles, demande quel test le nombre maximum d'inconnues des systemes calculables avec genre de machine.

M. Facre répond que la machine employé pour ic calcul des pertes d'énergie dans les marées permet do résoudre des systèmes d’équations à $15-20$ inconnues, mais qu'il existe des types permettant la solution de systèmes a 30 inconnues.

Cette capacité de calcul paraissant faible a M. le Président, pour les calculs de barrage divises en anneaux horizontaux et en murs, M. Meren signale un procede de subdivision des inconnues, qui est applicable pour la plupart des problemes avec conditions anx limites. Si on cherche par exemple $\Phi$ dans un domaine dans lequel $د \Phi=0$ et où $\Phi$ est connu sur le contour, on peut chev cher d'abord $\partial \mathrm{w} / \partial n$ sur le conlour, il n'y a alors qu'environ $3 n$ inconnus alors qu'il y a environ $n^{22}$ si on cherche directement $\Phi$ dans le domaine - on en deduit alors क partout par une formule de Green.

M. Meren signale dautre part que los erreurs faifos sur les problèmes avec conditions aux limites sont plus petites que celles qui entachent les problemes arec conditions initiales: dans les premiers, en effet, par excmple avec l'équation de Laptacr, les phénomènes ont tendance $\grave{a}$ s'égaliser à l'intérieur des limites, tandis que dans les autres, la moindre erreur s'amplifie au fur et à mesure des opérations. Il résulte de l'ensemble de ces remarques que les machines à eartes perforées paraissent pouvoir rendre beaucoup de services en hydraulique.

M. le Président remarque qu'en elasticité, où les problemes presentent des conditions aux limites, la conclusion de M. Meyen est rassurante.

La séance est levée à 11 h 46 . 